\title{
AVALIAÇÃO IN VITRO DO POTENCIAL ANTIMICROBIANO DO ÓLEO ESSENCIAL DE Lippia ssp FRENTE A LEVEDURAS DE OCORRÊNCIA NA CANDIDÍASE BUCAL. \\ Horácio Freitas Bomfim ${ }^{1}$; Angélica Maria Lucchese ${ }^{2}$; Lucciano Brandão de Lima ${ }^{3}$ \\ 1. Bolsista PIBIC/CNPq, graduando em Farmácia , Universidade Estadual de Feira de Santana, e-mail: hfreitasb@gmail.com 2. Orientador, Departamento de Exatas, Universidade Estadual de Feira de Santana, e-mail: angélica.lucchese@gmail.com 3. Co-orientador, Programa de Pós-graduação em Biotecnologia, Universidade Estadual de Feira de Santana, e-mail: lucciano.odonto@gmail.com
}

PALAVRAS-CHAVE: Candida; Lippia spp; óleo essencial.

\section{INTRODUÇÃO}

A candidíase bucal caracteriza-se pelo aparecimento de lesões superficiais ou profundas, agudas ou crônicas (CLIFF, et al., 2008) podendo ser causada por diferentes espécies do gênero Candida, entre elas, C. albicans, C. tropicalis, C. glabrata, C. krusei, C. parapsilosis, C. guilliermondii (ZEGARELLI, 1993). Trata-se de uma infecção oportunista que acomete pacientes hospitalizados, principalmente em unidades de terapia intensiva, assim como indivíduos imunosuprimidos (SIQUEIRA, et al., 2014). O tratamento consiste no uso de antifúngicos tópicos a exemplo da nistatina, ou na administração sistêmica de medicamentos como o fluconazol e a anfotericina-B (NETO; DANESI; UNFER, 2008).

A busca por novas substâncias de interesse farmacológico presentes em plantas envolve uma série de fatores, dentre eles o uso popular e a presença de metabólitos secundários com atividade biológica já comprovada (CALIXTO, 2005). O gênero Lippia tem uma grande importância para a flora brasileira e neotropical devido a sua representatividade e utilização econômica, como condimento e pelas suas propriedades medicinais (REIS, et al., 2014). Estudos farmacológicos das espécies de Lippia comprovam sua atividade antimicrobiana, antifúngica, larvicida e repelente, com destaque para os óleos essenciais (COSTA et al., 2017). Assim, notou-se a importância de avaliar a atividade antifúngica frente as espécies C. albicans, C. parapsilosis, C. glabrata, C. krusei e C. tropicalis dos óleos essenciais das espécies de Lippia.

\section{METODOLOGIA}

Os óleos essenciais dos três quimiotipos de L. origanoides (quimiotipos ricos em carvacrol - LOC, em timol - LOT e linalol - LOL), bem como de L. lasiocalycina (rico em $\beta$ mirceno e E-ocimenona - LL) e L. insignis (rico em E-ocimenona e limoneno - LI) foram cedidos pelo Laboratório de Química de Produtos Naturais e Bioativos (LAPRON/UEFS).

Foram testadas cepas padrão de C. albicans (ATCC 10231), C. parapsilosis (ATCC 22019), C. krusei (ATCC 14243), C. tropicalis (ATCC 13803) e C. glabrata (ATCC 36909) cedidas pelo do Laboratório de Pesquisa em Microbiologia (LAPEM/UEFS). 
Para a preparação do inóculo foram transferidas colônias dos micro-organismos teste isoladas da placa contendo ágar Sabouraud dextrose (ASD), com 24 h de crescimento, para um tubo de vidro de $5 \mathrm{~mL}$ contendo $2 \mathrm{~mL}$ de solução salina a $0,45 \%$ estéril. Após a homogeneização por $15 \mathrm{~s}$, a densidade do inóculo foi padronizada e ajustada a uma turbidez óptica da solução padrão na escala 0,5 de Mac Farland, que corresponde a aproximadamente 1 x $10^{6} \mathrm{UFC} / \mathrm{mL}$. Em seguida, realizou-se uma diluição 1:100 dessa suspensão em solução salina estéril a $0,45 \%$, obtendo a suspensão de trabalho com concentração de $1 \times 10^{4}$ $\mathrm{UFC} / \mathrm{mL}$. Este procedimento foi realizado para cada levedura testada isoladamente.

Para o preparo das placas de determinação da concentração inibitória mínima (CIM), $90 \mu \mathrm{L}$ de caldo Sabouraud dextrose (CSD) estéril duas vezes concentrado foram colocados em placas com 96 poços, na linha A1-A9 e na linha F10-F12 para controle de esterilidade do meio. Posteriormente, foram adicionados $90 \mu \mathrm{L}$ de CSD uma vez concentrado nas linhas B1B9, C1-C9, D1-D9, E1-E9, F1-F9, G1-G9, H1-H9 e na linha E10-E12 sendo esta última utilizada para controle de esterilidade do meio. Em seguida, foram adicionados $90 \mu \mathrm{L}$ da emulsão dos óleos essenciais a 1\% (em Tween 80 à 5\%) nas linhas A1-A9, produzindo uma concentração inicial de 0,5\%. Foram feitos controles para avaliar a esterilidade dos meios de cultura e dos óleos e para comprovar a viabilidade dos micro-organismos.

Em seguida, realizou-se a misturas dos óleos com o meio de cultura e alíquotas de 90 $\mu \mathrm{l}$ foram transferidas para cada um dos poços até o final da placa. Posteriormente, foram adicionados $10 \mu \mathrm{l}$ da suspensão do micro-organismo, perfazendo um volume final de 100 $\mu \mathrm{l} /$ poço, com uma concentração final de $1 \times 10^{3} \mathrm{UFC} / \mathrm{ml}$. Tween 80 a $5 \%$ foi avaliada para verificar se apresentava atividade frente aos micro-organismos. Nistatina $1 \%$ foi usada como controle positivo. As placas foram incubadas a $37^{\circ} \mathrm{C}$ por $48 \mathrm{~h}$. Após o período de incubação foram adicionados em cada poço $30 \mu \mathrm{l}$ do revelador Resazurina $0,01 \%$ (v/v). A leitura das placas foi feita após 3 horas de incubação a fim de determinar a CIM. A mudança de cor do revelador de azul para rosa/vermelho indicou crescimento microbiano.

Para determinação da concentração fungicida mínima (CFM), placas de Petri contendo ASD foram divididas em pequenos quadrantes e identificadas de acordo com a numeração dos poços das placas de CIM que inibiram o crescimento das leveduras. Nestes quadrantes foram inoculados $10 \mu 1$ do conteúdo de cada poço correspondente e estas seguiram para incubação a $37^{\circ} \mathrm{C}$ por 24 horas. A CFM foi considerada a menor concentração que impediu o crescimento visível neste subcultivo. Todos os testes foram realizados em triplicata. 
A tabela 01 apresenta os resultados de determinação da CIM dos óleos essenciais.

Tabela 01: Determinação das Concentrações Inibitórias Mínimas (CIM) dos óleos essenciais

\begin{tabular}{|c|c|c|c|c|c|c|c|}
\hline \multirow[t]{2}{*}{ CEPAS } & \multicolumn{7}{|c|}{$\begin{array}{c}\text { CIM } \\
\text { ÓLEOS ESSENCIAIS/CONTROLES }\end{array}$} \\
\hline & LOC & LOT & $\begin{array}{c}\mathbf{L O} \\
\mathbf{L}\end{array}$ & LI & $\mathbf{L L}$ & Tween 5\% & Nistatina \\
\hline C. albicans & $\begin{array}{c}0,12 \% \\
(1,2 \mathrm{mg} / \mathrm{mL})\end{array}$ & $\begin{array}{c}0,25 \% \\
(2,3 \mathrm{mg} / \mathrm{mL})\end{array}$ & $\mathrm{R}$ & $\begin{array}{c}0,12 \% \\
(1,1 \mathrm{mg} / \mathrm{mL})\end{array}$ & $\begin{array}{c}0,25 \% \\
(2,2 \mathrm{mg} / \mathrm{mL})\end{array}$ & $\mathrm{R}$ & $<0,6 \mathrm{mg} / \mathrm{mL}$ \\
\hline C. glabrata & $\begin{array}{c}0,06 \% \\
(0,59 \mathrm{mg} / \mathrm{mL})\end{array}$ & $\begin{array}{c}0,12 \% \\
(1,2 \mathrm{mg} / \mathrm{mL})\end{array}$ & $\mathrm{R}$ & $\begin{array}{c}0,06 \% \\
(0,59 \mathrm{mg} / \mathrm{mL})\end{array}$ & $*$ & $\mathrm{R}$ & $<0,6 \mathrm{mg} / \mathrm{mL}$ \\
\hline C. parapsilosis & $\begin{array}{c}0,06 \% \\
(0,59 \mathrm{mg} / \mathrm{mL})\end{array}$ & $\begin{array}{c}0,12 \% \\
(1,2 \mathrm{mg} / \mathrm{mL})\end{array}$ & $\mathrm{R}$ & $\begin{array}{c}0,12 \% \\
(1,1 \mathrm{mg} / \mathrm{mL})\end{array}$ & $\begin{array}{c}0,12 \% \\
(1,1 \mathrm{mg} / \mathrm{mL})\end{array}$ & $\mathrm{R}$ & $<0,6 \mathrm{mg} / \mathrm{mL}$ \\
\hline C. tropicalis & $\begin{array}{c}0,25 \% \\
(2,3 \mathrm{mg} / \mathrm{mL})\end{array}$ & $\mathrm{R}$ & $\mathrm{R}$ & $\begin{array}{c}0,25 \% \\
(2,2 \mathrm{mg} / \mathrm{mL})\end{array}$ & $*$ & $\mathrm{R}$ & $0,25 \mathrm{mg} / \mathrm{mL}$ \\
\hline C. krusei & $\begin{array}{c}0,5 \% \\
(4,7 \mathrm{mg} / \mathrm{mL})\end{array}$ & $\mathrm{R}$ & $\mathrm{R}$ & $\begin{array}{c}0,5 \% \\
(4,4 \mathrm{mg} / \mathrm{mL})\end{array}$ & $\mathrm{R}$ & $\mathrm{R}$ & $0,25 \mathrm{mg} / \mathrm{mL}$ \\
\hline
\end{tabular}

Todas as amostras testadas apresentaram atividade frente a pelo menos duas espécies de Candida, com exceção da espécie LOL, que não teve atividade frente a nenhuma das leveduras testadas. O óleo essencial de LOC apresentou o melhor desempenho frente às espécies de Candida testadas, com atividade frente à $C$. albicans, C. glabrata e $C$. parapsilosis com valores de CIM de $1,2 \mathrm{mg} / \mathrm{ml}, 0,59 \mathrm{mg} / \mathrm{ml}$ e $0,59 \mathrm{mg} / \mathrm{ml}$, respectivamente.

Duas das espécies de Candida (C. tropicalis e C. krusei) apresentaram maior resistência aos óleos testados e a nistatina, sendo ambas resistentes a LOT e LOL. O óleo essencial de $L L$ também não foi capaz de inibir o crescimento microbiano nas concentrações avaliadas frente $C$. krusei. A espécie $C$. glabrata foi a que apresentou menor resistência às emulsões testadas.

Os resultados da determinação da Concentração Fungicida Mínima (CFM) estão representados na tabela 02 .

Tabela 02: Determinação das Concentrações Fungicidas Mínimas (CFM), das emulsões de óleos essenciais de espécies de Lippia.

\begin{tabular}{|c|c|c|c|c|c|c|c|}
\hline \multirow[t]{2}{*}{ Candida SPP } & \multicolumn{7}{|c|}{$\begin{array}{c}\text { CFM } \\
\text { Óleos essenciais/controles }\end{array}$} \\
\hline & LOC & LOT & LOL & LI & $\mathbf{L L}$ & $\begin{array}{c}\text { Tween } \\
5 \%\end{array}$ & Nistatina \\
\hline C. albicans & $0,5 \%$ & $>0,5 \%$ & $\mathrm{R}$ & $0,5 \%$ & $>0,5 \%$ & $\mathrm{R}$ & $<0,6 \mathrm{mg} / \mathrm{mL}$ \\
\hline C. glabrata & $0,25 \%$ & $0,5 \%$ & $\mathrm{R}$ & $0,5 \%$ & $*$ & $\mathrm{R}$ & $<0,6 \mathrm{mg} / \mathrm{mL}$ \\
\hline C. parapsilosis & $0,25 \%$ & $0,5 \%$ & $\mathrm{R}$ & $>0,5 \%$ & $>0,5 \%$ & $\mathrm{R}$ & $<0,6 \mathrm{mg} / \mathrm{mL}$ \\
\hline C. tropicalis & $>0,5 \%$ & $\mathrm{R}$ & $\mathrm{R}$ & $>0,5 \%$ & $*$ & $\mathrm{R}$ & $0,25 \mathrm{mg} / \mathrm{mL}$ \\
\hline C. krusei & $>0,5 \%$ & $\mathrm{R}$ & $\mathrm{R}$ & $>0,5 \%$ & $\mathrm{R}$ & $\mathrm{R}$ & $0,25 \mathrm{mg} / \mathrm{mL}$ \\
\hline
\end{tabular}


R (resistente); * (não testada); LOC (L. origanoides rica em carvacrol); LOT (L. origanoides rica em timol); LOL (L. origanoides rica em linalol); LI (L. insignis); LL (L. lasiocalycina)

Os óleos testados apresentaram atividade fungistática frente à espécie C. albicans nas concentrações determinadas nos testes de CIM, sendo necessárias concentrações no mínimo duas vezes maiores que a concentração inibitória mínima para ter uma ação fungicida.

Os constituintes majoritários como carvacrol, timol e limoneno são substâncias com conhecida atividade antimicrobiana e comumente encontradas nos óleos essenciais de Lippia (COSTA, 2017), entretanto a contribuição das substâncias minoritárias presentes não pode ser descartada devido ao efeito sinérgico.

\section{CONSIDERAÇÕES FINAIS}

O óleo essencial do quimiotipo de L. origanoides rico em carvacrol e o de L. insignis foram os mais ativos frente as cepas de Candida. Já o óleo essencial de L. origanoides rico em linalol foi o único que não apresentou atividade antifúngica frente a nenhuma das cepas avaliadas. Como os óleos essenciais dos quimiotipos de L. origanoides apresentaram variações na sua atividade antimicrobiana, é importante destacar a importância de se conhecer os quimiotipos da região, em especial de espécies utilizadas pela população, pois a modificação da composição química pode influenciar na atividade biológica.

\section{REFERÊNCIAS}

CAVALCANTI Y W, ALMEIDA L F D, PADILHA W W N. Atividade Antifúngica de Três Óleos Essenciais Sobre Cepas de Candida. Rev Odontol Bras Central; 2011.

CLIFF, P. R, et al. Use of multilocus sequence typing for the investigation of colonisation by Candida albicans in intensive care unit patients. Journal of Hospital Infection p. 24, 2008.

CLSI. Methods for Dilution Antimicrobial Susceptibility Tests for Bacteria That Grow Aerobically; Approved Standard-6 Ed. CLSI document M7-A6 (ISBN 1- 56238-486-4). CLSI, 940 West Valley Road, Suite 1400, Wayne, Pennsylvania 19087- 1898 USA. 2003.

COSTA, P. S., et al. Atividade antimicrobiana e potencial terapêutico do gênero Lippia sensu lato (Verbenaceae). Hoehnea, v. 44, n. 2, p. 158-171, 2017.

NETO, M. M; DANESI, C. C.; UNFER, D. T. Candidíase Bucal. Saúde. v. 31, p. 16-26, 2005.

REIS, A. C., et al. Lippia alba (Verbenaceae): A New Tropical Autopolyploid Complex. American Journal of Botany, p. 1002-1012, 2014.

SIQUEIRA, J. da S. S., et al. Candidíase oral em pacientes internados em UTI. Revista Brasileira de Odontologia. Rio de Janeiro, v. 71, n. 2, p. 176-9, 2014.

ZEGARELLI, D. Fungal infections of the oral cavity. Otolaryngologic Clinics of North America 26:1069-1088, 1993. 